\title{
Das „Christian Doppler Labor für Sedimentforschung und -management": Anwendungsorientierte Grundlagen- forschung und Herausforderungen für eine nachhaltige Wasserkraft und Schifffahrt
}

\author{
Christoph Hauer · Beatrice Wagner · Johann Aigner · Patrick Holzapfel · Peter Flödl · Marcel Liedermann · \\ Michael Tritthart · Christine Sindelar · Mario KIösch · Marlene Haimann · Helmut Habersack
}

Online publiziert: 24. Januar 2019

(C) Der/die Autor(en) 2019, korrigierte Publikation 2019

\begin{abstract}
Zusammenfassung Das Christian Doppler Labor für Sedimentforschung und -management hat zum Ziel, (i) Möglichkeiten zur optimierten ökonomischen, technischen bzw. ökologischen Nutzung der Wasserkraft, insbesondere Minimierung der Stauraumverlandung, (ii) zur Verbesserung des Sedimentmanagements bei Wasserstraßen und (iii) zur Verlängerung der Lebensdauer unterschiedlicher technischer Anlagenteile von Wasserkraftanlagen (Einlaufbauwerke, Pumpen und Turbinen) $\mathrm{zu}$ erarbeiten. Wesentlich im Projekt sind die Reduktion der Kosten und ein verbessertes Sedimentmanagement, sowohl in alpinen als auch in industriell geprägten Flusslandschaften, unter Einhaltung technischer und ökologi-
\end{abstract}

PD DI Dr. C. Hauer $(\bowtie) \cdot$

DI B. Wagner · DI J. Aigner .

DI P. Holzapfel · DI P. Flödl, BSc. ·

DI Dr. M. Liedermann .

PD DI Dr. M. Tritthart .

DI M. Klösch · DI M. Haimann .

Univ.-Prof. DI Dr. H. Habersack

Department für

Wasser-Atmosphäre-Umwelt,

Christian Doppler Labor

für Sedimentforschung

und -management, Institut

für Wasserbau, Hydraulik

und Fließgewässerforschung,

Universität für Bodenkultur Wien,

Muthgasse 107, 1190 Wien, Österreich

christoph.hauer@boku.ac.at

DI Mag. Dr. C. Sindelar

Department für

Wasser-Atmosphäre-Umwelt,

Christian Doppler Labor

für Sedimentforschung

und -management, Institut

für Wasserbau, Hydraulik

und Fließgewässerforschung,

Universität für Bodenkultur Wien,

Muthgasse 18, 1190 Wien, Österreich scher Vorgaben. Aufbauend auf dem Stand des Wissens wird im CD-Labor anwendungsorientierte Grundlagenforschung in Bezug auf Erosion, Transport, Sedimentation und Remobilisierung von Feststoffen durchgeführt. Im Zuge der wissenschaftlichen Arbeiten ist eine Kombination von Laborversuchen (z.B. physikalische Modellversuche) und Felduntersuchungen (z. B. Monitoring von Speicherentlandungen) auf unterschiedlichen Skalen ( $\mu \mathrm{m}$ bis Einzugsgebiet) vorgesehen, um ein verbessertes Prozessverständnis zu erzielen. Weiters werden durch Entwicklung oder Adaptierung von hydrodynamisch-numerischen Modellen und innovativen Anwendungen im Bereich des Monitorings (z.B. seismic profiling), neue technische Standards für die nationale und internationale Wasserkraftindustrie erarbeitet.

\section{Schlüsselwörter}

Stauraummanagement - Wasserkraft .

Verlandung · Sedimentmonitoring und -modellierung · Physikalischer Modellversuch · Prozessverständnis

The "Christian Doppler

Laboratory for Sediment

Research and Management": application-oriented basic research and challenges for sustainable hydropower

Abstract The Christian Doppler Laboratory for Sediment Research and Management aims to establish and preserve the long-term use of reservoir capacities and a long technical lifespan of various plant components, such as intake channels, pumps and turbines. Moreover, the preservation of given flood protection (due to reservoirs) like peak flow retention and the important flood storage capacity will be targeted.
Module 1 aims to improve the economical, technical and ecological standards for hydropower use. Module 2 focuses on the improvement of the sediment management for industrialised rivers, and Module 3 targets to achieve a long technical life span of various plant components. However, all these issues will be addressed within the framework of the overall aim to minimise the costs in future by improved sediment management in alpine regions and industrialised river catchments. Based on the determination of the state-ofthe-art engineering practice, application-oriented basic research will be conducted concerning erosion, transport, sedimentation and remobilisation of bedload and suspended sediments. Here, a combination of both laboratory (e.g. flume experiments, physical models) and field studies (e.g. monitoring of reservoir flushing) on different scales ( $\mu \mathrm{m}$ - catchment) is expected to enable an improved process understanding. Moreover, based on the development or adaptation of hydrodynamic-numerical models and monitoring techniques (e.g. seismic profiling), advanced tools will be provided for the national and international hydropower industry.

Keywords Reservoir management . Hydropower · Sedimentation . Sediment monitoring and modelling . Physical laboratory study · Process understanding

\section{Einleitung}

Prognosen zeigen, dass in den nächsten 20 Jahren $60 \%$ aller Investitionen im Energiesektor im Bereich der erneuerbaren Energieerzeugung liegen. Vorhersagen in Bezug auf den Ausbau der Wasserkraft weisen hier einen Erzeugungsanteil von $25 \%$ aller zukünf- 
tigen erneuerbaren Energieträger auf, vor allem aufgrund des Potenzials und der Ausbaupläne in China, Afrika, Lateinamerika und Südostasien. Auch in Europa wird eine Zunahme des Wasserkraftanteils an der Energieproduktion aufgrund der Emissionsziele der Europäischen Union bis 2050 angestrebt bzw. gefördert (European Commission 2018). Eine der wesentlichen Herausforderungen der Wasserkraftnutzung in Bezug auf den ökonomischen und technischen Betrieb sowie die ökologische Verträglichkeit (gefordert durch die Europäische Wasserrahmenrichtlinie) ist die Entnahme, notwendige Deponierung und gestörte Dynamik von Sedimenten in Flusseinzugsgebieten, welche das zukünftige Marktpotenzial der Wasserkraftindustrie bedeutend einschränken wird. Aufgrund eines fehlenden Bewusstseins (bzw. fehlenden Prozessverständnisses) in Bezug auf die Notwendigkeiten eines nachhaltigen Sedimentmanagements zeigen sich (sehr deutlich) unterschiedliche ökonomische, technische und ökologische Probleme, die sowohl die Wasserkraftindustrie und die zuständigen Behörden betreffen, aber auch gesellschaftliche Aspekte (soziale Akzeptanz) beinhalten.

Das CD-Labor „Sedimentforschung und -management" hat zum Ziel, Möglichkeiten zur optimierten ökonomischen, technischen bzw. ökologischen Nutzung der Wasserkraft (Modul 1), zur Verbesserung des Sedimentmanagements bei Wasserstraßen (Modul 2) und zur Verlängerung der Lebensdauer unterschiedlicher technischer Anlagenteile von Wasserkraftanlagen (Einlaufbauwerke, Pumpen und Turbinen) (Modul 3) zu erarbeiten. Weiters ist die Gewährleistung der Hochwassersicherheit (durch Speicher) mittels Reduktion der Spitzenabflüsse und Bereitstellung von Retentionsvolumen unter Berücksichtigung ökologischer Kriterien ein Ziel der geplanten Arbeiten. Oberstes Ziel des CD-Labors ist die Reduktion der Kosten und ein verbessertes Sedimentmanagement, sowohl in alpinen als auch in industriell geprägten Flusslandschaften, unter Einhaltung technischer und ökologischer Vorgaben. Aufbauend auf dem Stand des Wissens wird im CD-Labor anwendungsorientierte Grundlagenforschung in Bezug auf Erosion, Transport, Sedimentation und Remobilisierung von Feststoffen durchgeführt. Für die Durchführung ist eine Kombination von Laborversuchen
(z.B. physikalische Modellversuche) und Felduntersuchungen (z. B. Monitoring von Speicherentlandungen) auf unterschiedlichen Skalen ( $\mu \mathrm{m}$ bis Einzugsgebiet) vorgesehen, um ein verbessertes Prozessverständnis zu erzielen. Weiters werden durch Entwicklung oder Adaptierung von hydrodynamisch-numerischen Modellen sowie innovativen Anwendungen im Bereich des Monitorings (z. B. „Seismic Profiling“) neue technische Standards für die nationale und internationale Wasserkraftindustrie erarbeitet.

Das CD-Labor „Sedimentforschung und -management" ist am Department für Wasser - Atmosphäre - Umwelt an der Universität für Bodenkultur Wien situiert und hat im Oktober 2017 unter Begleitung durch die vier Unternehmenspartner Verein für Ökologie und Umweltforschung (VÖU), viadonau, Andritz AG und Voith GmbH die Forschungsarbeit aufgenommen. Der VÖU als Vertreter der Wasserkrafterzeugung ist Partner des Moduls 1. Viadonau beteiligt sich aufgrund ihrer Verantwortung in Bezug auf die Schiffbarkeit der Donau an den Untersuchungen des Moduls 2. Im Modul 3 unterstützen Andritz AG und Voith $\mathrm{GmbH}$ die Arbeiten zur Verlängerung der Lebensdauer unterschiedlicher technischer Anlagenteile im Bereich der Turbinen. Weiters ist geplant, zu einem späteren Zeitpunkt ein zusätzliches Modul (Untersuchungen von Abrasion) in das CDLabor einzugliedern.

Die Ergebnisse des CD-Labors werden nicht nur zur Definition von neuen Standards im Bereich der ökonomischen, technischen und ökologischen Optimierung der Wasserkraftnutzung beitragen. Es sind auch generelle, innovative Konzepte in Bezug auf ein nachhaltiges Sedimentmanagement in industrialisierten Flusseinzugsgebieten durch (i) ein erweitertes Prozessverständnis, (ii) Erkenntnisse über Wechselwirkungen mit der Ökologie und (iii) Entwicklung von neuen Monitoring- bzw. Modellierungstechnologien $\mathrm{zu}$ erwarten. Weiters ist die Implementierung der Ergebnisse des CD-Labors „Sedimentforschung und -management" in Gewässerbewirtschaftungsplänen, Richtlinien und spezifischen Gesetzgebungen in Abstimmung mit VertreterInnen der Wasserbauverwaltung bzw. Wildbach- und Lawinenverbauung vorgesehen. Ein Schwerpunkt des vorliegenden Fachbeitrags liegt auf dem Stand der Technik und den Her- ausforderungen des Sedimentmanagements im Bereich von Wasserkraftanlagen (Modul 1).

\section{Sedimentmanagement bei Wasserkraftwerken}

Maßnahmen im Bereich des Sedimentmanagements von Wasserkraftwerken sind vielfältig und können grundsätzlich in drei Kategorien eingeteilt werden: (a) Maßnahmen im Einzugsgebiet, (b) Maßnahmen im Stauraum und (c) Maßnahmen am Staudamm/ Staubauwerk (Schleiss und Oehy 2002). Eine andere Klassifizierung unterscheidet zwischen Ansätzen zur (i) Verringerung des Sedimenteintrags in Speicher, (ii) Verhinderung bzw. Minimierung der Verlandung durch Umleitung der Sedimente um bzw. durch Stauräume sowie jenen zur (iii) Erhöhung oder Rückgewinnung des Speichervolumens durch Entfernung von Sedimentablagerungen in den Stauräumen (Kondolf et al. 2014). Einen Überblick über bestehende Maßnahmen verschafft Abb. 1.

Die Maßnahmen zum Sedimentmanagement auf Einzugsgebietsebene werden im Allgemeinen als präventive Maßnahmen eingestuft und erstrecken sich von Maßnahmen zum natürlichen Sedimentrückhalt infolge erosionshemmender, hangstabilisierender Bepflanzungen sowie einer angepassten Landnutzung und -bewirtschaftung über Maßnahmen zum technischen Feststoffrückhalt (z. B. Geschieberückhaltebecken) bis hin zu Wasserüberleitungen aus Stauseen in benachbarte Einzugsgebiete. Als Maßnahme im Einzugsgebiet sowie im Speicher zählen Umleit- und Spülstollen (sog. SedimentBypass-Systeme) (Boes 2011; Schleiss et al. 2010), die das sedimentbeladene Wasser am Speicher vorbei oder durch den Speicher leiten (Abb. 2). Eine der größten Herausforderungen im Zusammenhang mit Sedimentumleitstollen, insbesondere bei einem hohen Anteil an hartem Gestein wie Quarz oder Feldspat, ist die Abrasion des Tunnelbodens infolge des transportierten Sediments (Sumi et al. 2004).

Andere häufig angewandte Maßnahmen im Stauraum und am Staubauwerk können als jene Technologien zusammengefasst werden, dessen Ziel es ist, Sedimente aus dem Stauraum $\mathrm{zu}$ entfernen. Dies erfolgt entweder über eine mechanische (Trocken- oder Nassbaggerung) oder hydraulische Räumung (Stauraumspülung) (Gaisbaucher 


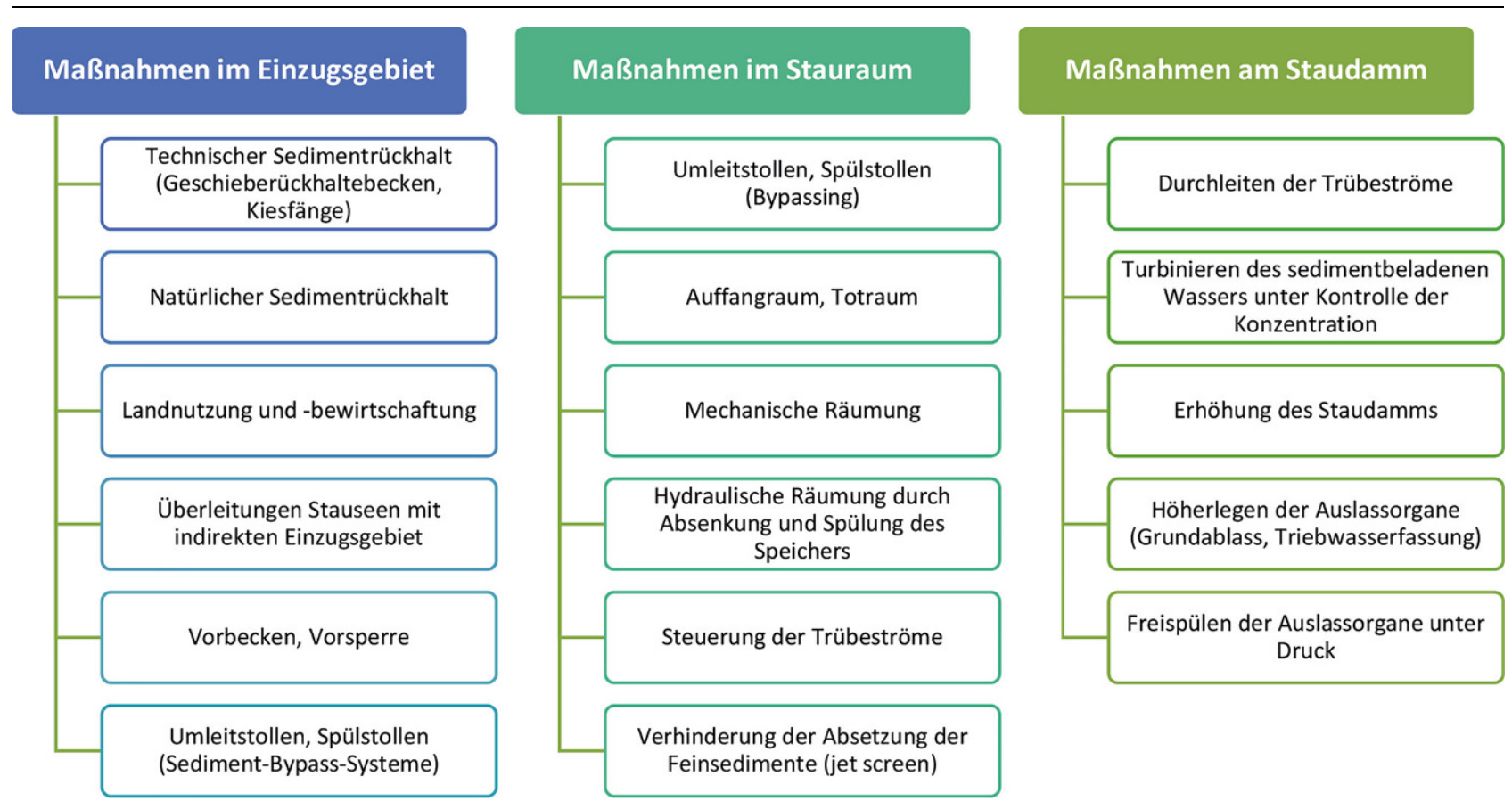

Abb. 1 Maßnahmenzur Bewältigung ökonomischerund technischer Herausforderungen durch Stauraumverlandung (Wagner et al. 2013; nach Schleiss et al. 2010)
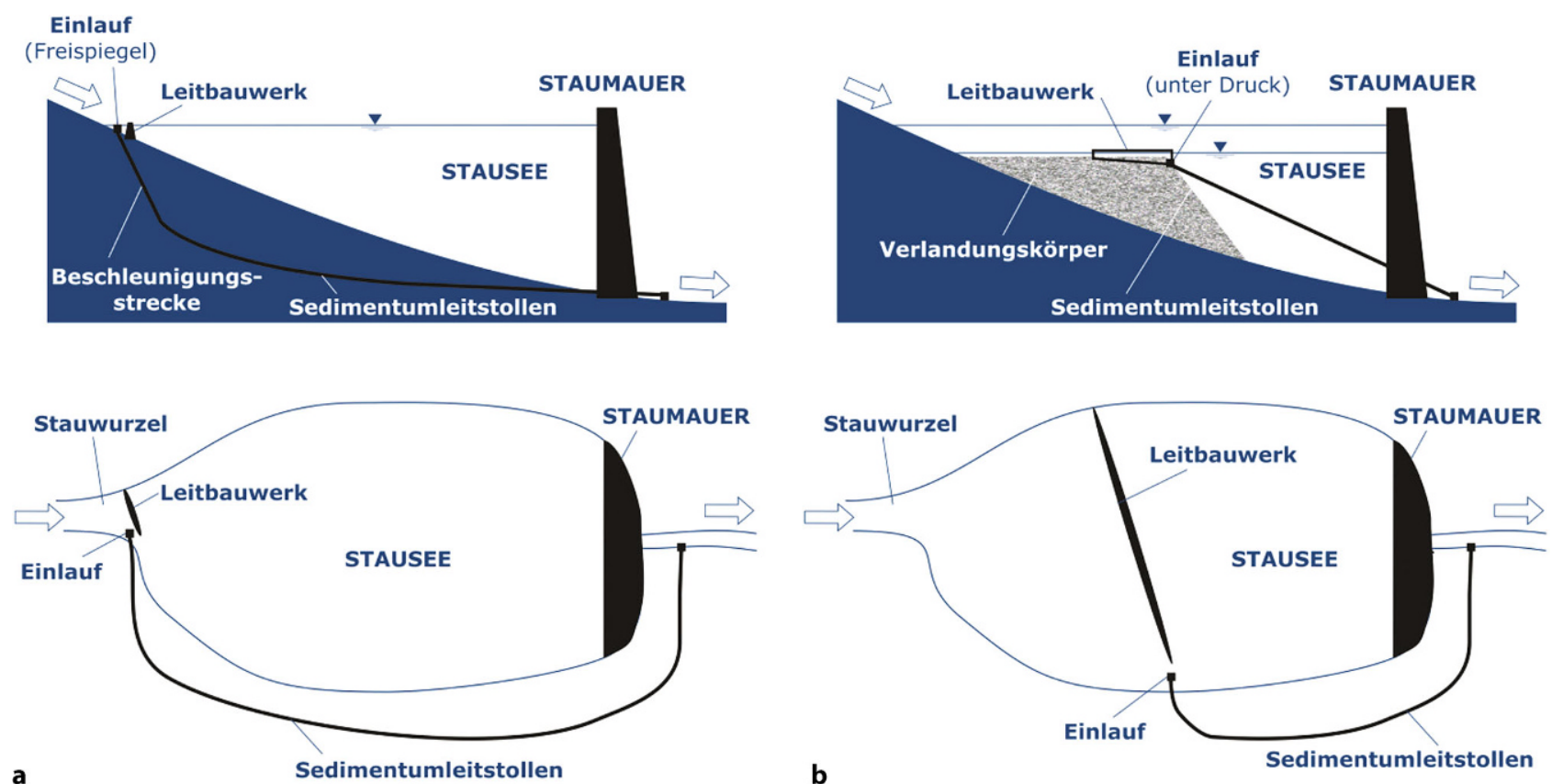

Abb. 2 SchematischeDarstellung eines Sedimentumleitstollens. Lage des Einlaufbereichs (a) bei der Stauraumwurzel, (b) innerhalb des Stauraums (nach Boes 2011)

und Knoblauch 2001; Mamede 2008). Darüber hinaus existieren auf Stauraumebene zahlreiche Maßnahmen zur Steuerung der Trübeströme (stark sedimentbeladene Wassermassen) sowie Verhinderung der Absetzung von Feinsedimenten. Auf der Ebene des Staubauwerks werden Möglichkeiten zur Durchleitung von Trübeströmen sowie Technologien zum möglichst schadlosen Turbinieren des sedimentbeladenen Wassers unter Kontrolle der Konzentration zusammengefasst. Im Allgemeinen zielen die entwickelten Technologien darauf ab, Trübeströme entweder durch den Stauraum zu leiten oder zu stoppen, eine Sedimentation an kritischen Punkten zu verhindern oder Trübeströme $\mathrm{zu}$ verdünnen und sie durch den Staudamm zu transportieren. Als technische Lösungen werden hier u. a. undurchlässige und durchlässige Barrieren wie Dämme und Geotextilvorhänge oder hydraulische Geräte 


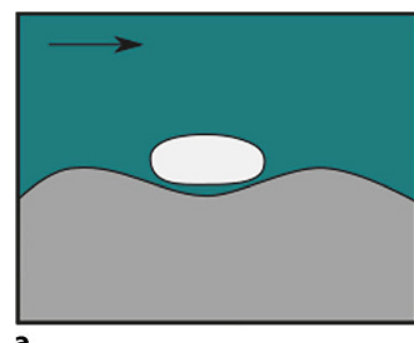

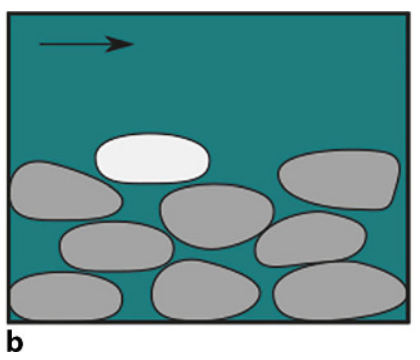
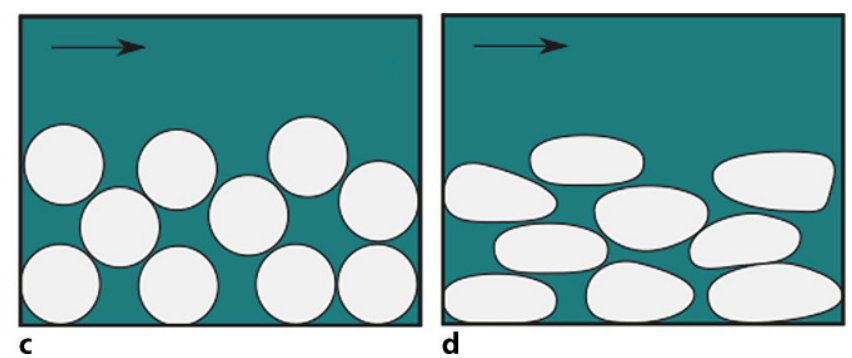

Abb. 3 Darstellung der verschiedenen Setups für numerische Simulationen; dunkelgraue Objekte unbeweglich; hellgraue Objekte frei beweglich

(,jet screens") verwendet (De Cesare et al. 2006; Schleiss et al. 2010). Weiters zählen Maßnahmen wie eine Erhöhung des Staudamms oder eine Höherlegung der Auslassorgane zu konstruktiven Maßnahmen auf Bauwerksebene. Die Wirksamkeit dieser Maßnahmen ist jedoch zeitlich begrenzt, da sie lediglich eine konstruktive Vergrößerung des Stauraumvolumens herbeiführen und keine nachhaltige Verringerung der Stauraumverlandung erzielen (Gaisbacher und Knoblauch 2001).

Betreffend den Stand der Technik im Sedimentmanagement lassen sich folgende Wissenslücken feststellen:

- Ein verbessertes Prozessverständnis zu Sedimentdynamik (Bewegungsbeginn, Transport, Ablagerung, Remobilisierung) ist eine wesentliche Grundlage für darauf aufbauende Forschungsarbeiten (z.B. hydrodynamisch-numerische Modellierung, physikalischer Modellversuch) und umfasst u.a. (i) mögliche Wechselwirkungen mit biologischen Prozessen (z. B. Biofilmen), (ii) Turbulenzmerkmale auf verschiedenen Skalen, (iii) realistische physikalische Modelle mit unterschiedlich geformten Steinen, (iv) Reynolds-induzierte Skalierungsfehler und (v) numerische Modelle mit hoher Auflösung betreffend Morphodynamik. Weiters kann das Prozessverständnis zur Seitenerosion verbessert werden; hier bestehen u. a. Wissensdefizite bezüglich der Konsolidierung heterogener Sedimente (Sand, Schluff) in Zusammenhang mit Stauraumspülungen („clustered sediment erosion“).

- Wesentliche Wissensdefizite im Zusammenhang mit dem Erreichen eines nachhaltigen Sedimentmanagements in Stauräumen von Wasserkraftwerken betreffen (i) die zunehmende Sedimentation des Stauraums durch fehlendes/unzureichendes Sedimentmanagement, (ii) Be- schädigung und Verringerung der Funktionsfähigkeit bestimmter technischer Elemente (z.B. Turbine), (iii) Erzeugungsverluste, Verringerung der Versorgungssicherheit sowie Verringerung des Hochwasserschutzes durch zunehmende Sedimentation im Stauraum und (iv) kontinuierlich hohe Kosten für die Räumung des verlandeten Sediments sowie die Behandlung insbesondere der feinen Anteile.

- Wissens- bzw. Informationsdefizite können ebenfalls im Bereich der Implementierung in die Gesetzgebung festgestellt werden. Diese betreffen beispielsweise den Wissensaustausch zwischen verschiedenen nationalen Bundesbehörden oder rechtliche Unsicherheiten bezüglich der Verantwortung (Kosten) in Bezug auf (Fein-)Sedimentablagerungen in Überschwemmungsgebieten.

\section{Numerische Modellierung des Sedimenttransports}

Hydrodynamisch-numerische Modelle weisen eine beträchtliche Zunahme in der Verwendung von geomorphologischen, hydrologischen und ökologischen Analysen auf (Hauer und Habersack 2009; Harby et al. 2005). Die Vorteile der numerischen Modellierung ergeben sich aus einem besseren allgemeinen Prozessverständnis sowie der Möglichkeit, Schlüsselprozesse durch Abbildung über mehrdimensionale Strömungsfelder zur Analyse eines Flusssystems oder Speichers zu simulieren.

Im Bereich von Stauräumen werden hydrodynamisch-numerische Modelle eingesetzt, um verschiedene Fragestellungen u.a. zu (i) Sedimenteintrag und -transport (siehe Abb. 3; Nutzung verschiedener Setups für numerische Simulationen), (ii) Trübeströmen (Strömungswege, Temperatur etc.), (iii) Stau- raumspülungen und (iv) technischen Elementen, wie Turbinen, Wehren oder Tauchbecken in Wechselwirkung mit Sediment zu untersuchen (An und Julien 2014; Haun et al. 2012; Mamede 2008; Young et al. 2005). Erste Arbeiten zur numerischen 3D-Modellierung und der damit verbundenen Transportprozesse in Stauräumen wurden von Tritthart (2000) durchgeführt. Dabei wurde die Reynolds-gemittelte Navier-StokesGleichung (RANS) gelöst und die FiniteVolumen-Methode zur Diskretisierung angewendet. Die Turbulenzschließung wurde mit einem k- $\varepsilon$-Modell durchgeführt und es wurde eine Variante des SIMPLE-Verfahrens (Patankar und Spalding 1972) zur Berechnung des nicht hydrostatischen Druckfelds angewendet. Das am Institut für Wasserbau, Hydraulik und Fließgewässerforschung entwickelte Sedimenttransportmodell (iSed) (Tritthart et al. 2011) ermöglicht eine Ermittlung des Transports von suspendiertem Sediment durch das Lösen einer Konvektions-DiffusionsGleichung. Dabei berechnet ein abschließender Modellierungsschritt die Kornsortierungsprozesse und ermittelt morphodynamische Änderungen der Stauraumsohle (siehe auch Tritthart et al., in diesem Heft). Dieses Modell wurde bereits für verschiedene Fragestellungen, z. B. zur Untersuchung der Sedimentationsprozesse in Speichern der Vorarlberger Illwerke oder zur Analyse der morphodynamischen Entwicklungen im Stauraum des Kraftwerks Feistritz an der Drau eingesetzt.

Im Bereich der numerischen Strömungsmechanik finden neben RANS auch andere hochauflösende Techniken, wie Direct Numerical Solutions (DNS) (Lin und Liu 1998), Large Eddy Simulations (LES) (Schlüter et al. 2005) und Smoothed Particle Hydrodynamics (Monaghan und Kos 1999) Anwendung, deren numerische Codes für detaillierte technische Studien und Environmen- 


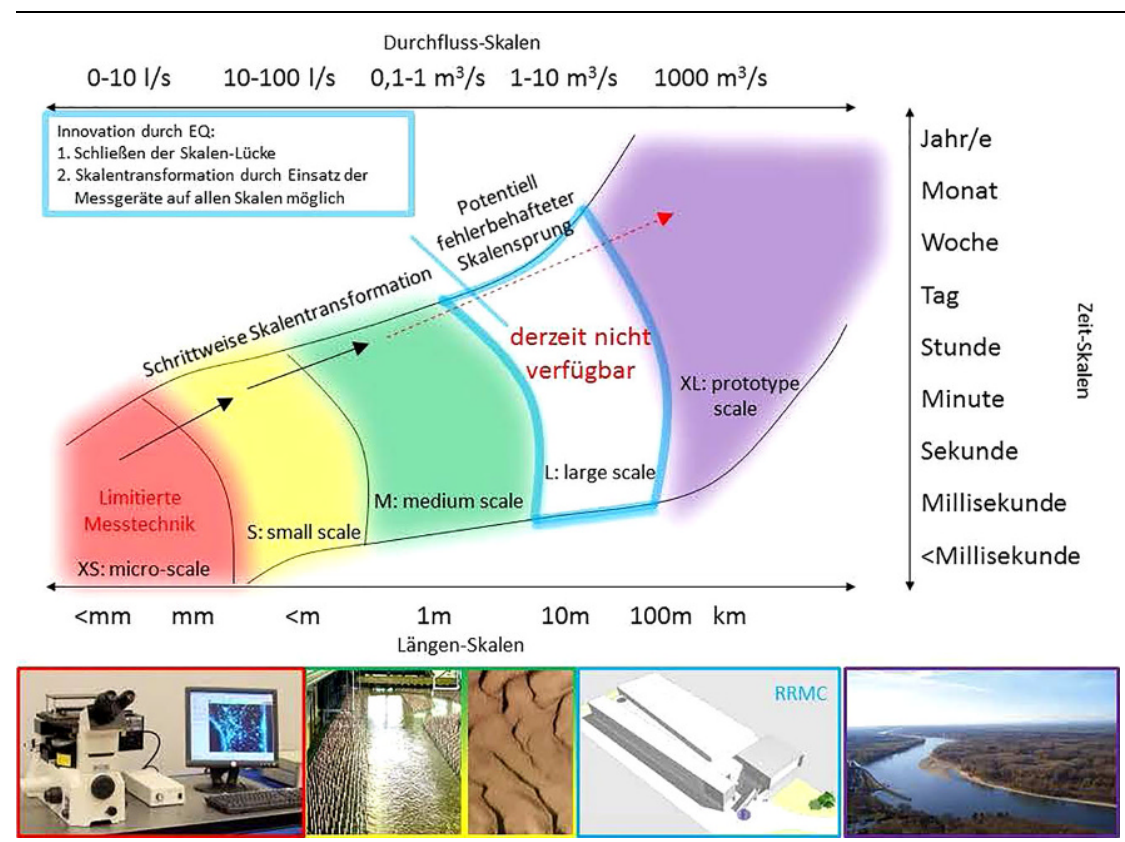

Abb. 4 Skalen in physikalischen Modellen und im Fließgewässer (,,nature scale“) und potenziell fehlerhafte Skalenlücke

tal-Flow-Analysen genutzt werden können, wie zum Beispiel Studien zu den Auswirkungen von Turbulenzphänomenen auf Makroinvertebraten.

Wissensdefizite im Bereich der Modellierung von Sedimenttransportprozessen resultieren aus Einschränkungen der empirischen Sedimenttransportgleichungen (häufig über- und unterschätzende Transportraten). Da keine vollständige mathematische Beschreibung vorliegt, können viele physikalisch ablaufenden Prozesse in numerischen Modellen von Stauräumen durch vorhandene Codes nicht ausreichend dargestellt werden (z.B. Vernachlässigung des Porenwasserdrucks/der Kohäsion in bestehenden Modellen bei der Analyse der Seitenerosion bei Stauraumspülungen).

\section{Sedimentmonitoring an großen Fließgewässern}

Sedimenttransportprozesse sind von vielen Faktoren, wie beispielsweise unterschiedlichen fluvialen Verhältnissen und morphologischen Veränderungen abhängig (Ashmore und Rennie 2013). Insbesondere an großen, industrialisierten Fließgewässern ist das Wissen über Sedimenttransportvorgänge und daraus resultierenden morphologischen Prozessen wesentlich für die Herstellung/Bewahrung der Schiffbarkeit (erforderliche Wassertiefen etc.). genüber diesen den Vorteil, dass sie die reale Strömungssituation abbilden können. Infolge aktueller Fortschritte im Bereich der laseroptischen Messungen können mittlerweile Einblicke in die zeitlich und räumlich fluktuierenden und komplexen Strömungsstrukturen auf bisher nicht darstellbarer Detailebene untersucht werden. Der Nachteil physikalischer Modelle besteht darin, dass es sich in der Regel um skalierte Modelle handelt. Um das Modell entsprechend zu skalieren, werden bei der Planung und Gestaltung Ähnlichkeitsgesetze angewendet (Glas et al. 2012). Der Umgang mit beweglichen Sedimenten in skalierten physikalischen Modellen erfordert, dass die PartikelReynolds-Zahl $R e^{*}$ und die dimensionslose Schubspannung $\mathrm{Fr}^{*}$ im skalierten Modell und im Maßstab 1:1 gleich sind. Es ist jedoch nicht möglich, die Ähnlichkeit von $\operatorname{Re}^{*}$ und $\mathrm{Fr}^{*} \mathrm{zu}$ erfüllen, ohne das Modell zu verzerren. Eine andere Möglichkeit besteht darin, sicherzustellen, dass $R e^{*}$ einen bestimmten Schwellenwert überschreitet, sodass $\mathrm{Fr}^{*}$ und somit die beginnende Bewegung von Sedimenten unabhängig von $R e^{*}$ ist (gemäß dem Shields-Diagramm).

Im Zusammenhang mit dem Sedimentmanagement von Wasserkraftwerken finden physikalische Modelle u.a. zur Untersuchung von Forschungsfragen $\mathrm{zu}$ (i) Trübeströmen (Yu et al. 2000), (ii) Sedimentumleitstollen (sog. Sediment-Bypass-Systeme) (Auel et al. 2011) oder (iii) Stauraumspülungen (Kantoush und Schleiss 2009) Anwendung. Im Rahmen des CD-Labors steht v.a. die Skalenlücke „nature scale“ im großmaßstäblichen Modellbereich im Mittelpunkt der Forschungsarbeiten (Abb. 4).

Um diese Skalenlücke zu schließen, werden im Rahmen des CD-Labors für Sedimentforschung und -management im neuen BOKU-Wasserbaulabor großmaßstäbliche Versuche gemacht, wo bis zu 10.0001/s ohne Pumpen zur Verfügung stehen werden.

\section{Turbinenabrasion} methodik zur Erfassung der Sedimentdynamik und (iii) unzureichende Auflösung der Wassertemperaturmessungen als grundlegende Information für die Analyse von Trübeströmen.

\section{Physikalische Modellversuche}

Physikalische Modelle sind von wesentlicher Bedeutung für die Kalibrierung numerischer Modelle und haben ge-
Turbinenausfälle reduzieren die nationale und internationale Sicherheit der Stromversorgung. Ein wesentliches Problem liegt im häufig unzureichenden Sedimentmanagement, wodurch sich insbesondere an Turbinen Abrasionserscheinungen bilden (Padhy und Saini 2009; Thapa et al. 2012) (Abb. 5). Sediment ist daher eine Herausforde- 


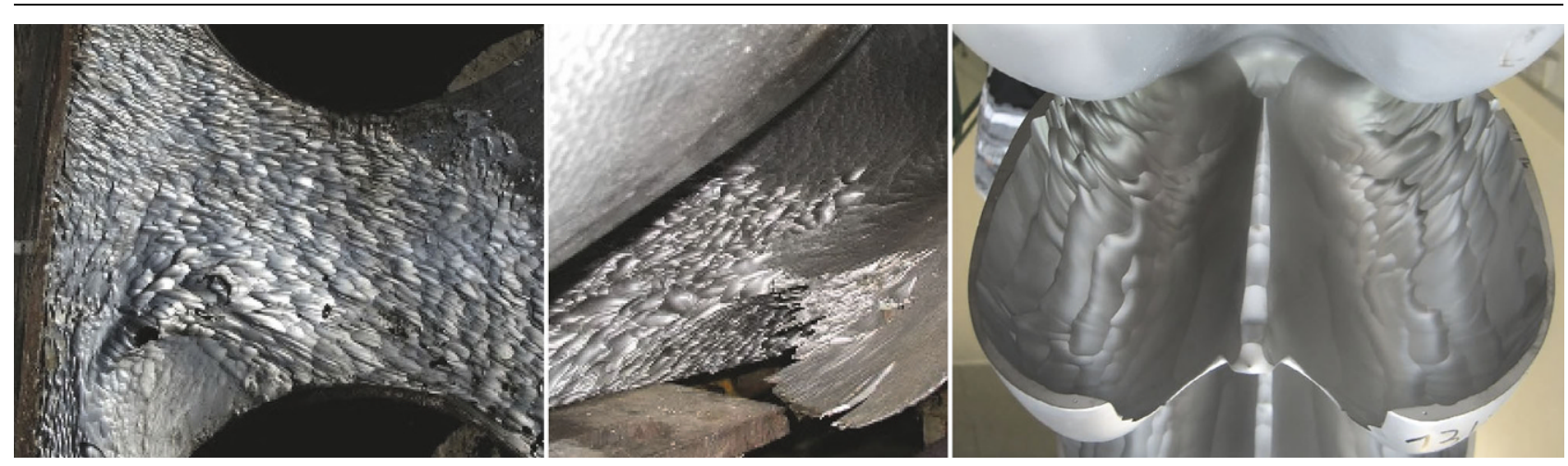

Abb. 5 Schäden an Turbinen infolge von Abrasion (Fotos: Voith, TIWAG)

rung für die Funktionalität der gesamten Wasserkraftanlage (Mamede 2008; Schleiss et al. 2010; Hauer et al. 2016a), da Abrasion neben einer Reduktion der Zuverlässigkeit der Stromversorgung auch negative Auswirkungen auf die Kosten der Kraftwerksbetreiber (z.B. Wartungskosten, Kostenverluste infolge von Betriebsunterbrechungen) hat (Neopane 2010). Im Allgemeinen ist das Abriebpotenzial abhängig von Faktoren wie Korngröße, Fließgeschwindigkeit, Härte und Konzentration (Padhy und Saini 2009). Dabei wird angenommen, dass Sedimentkörner, die eine kritische Größe $\left(\mathrm{d}_{\mathrm{cr}}\right)$ überschreiten, für Turbinenschäden verantwortlich sind (Truscott 1972). Darüber hinaus gibt es zur Zeit vertiefende Untersuchungen zur gewichteten Summe der Korngrößenverteilung in Zusammenhang mit dem Abriebpotenzial (Agrawal et al. 2011). Als wesentliche Faktoren für die $\mathrm{Ge}$ wichtung können hier Geschwindigkeit und Härte genannt werden.

Die Teilchengeschwindigkeit und -richtung sowie mehrere auf das Teilchen einwirkende Kräfte (die sich auf seinem Weg durch die Turbine stabilisieren und destabilisieren) werden durch die Strömung beeinflusst. Lysne et al. (2003) beschreiben zwei unterschiedliche Kraftkategorien: (i) „stabilising force" (viskose Kräfte und Schwerkräfte) und (ii) „destabilising force“ (Zentrifugalkraft, Coriolis-Kräfte, Turbulenzkraft). Daher muss ein besonderer Fokus darauf gelegt werden, sowohl (a) Fließeigenschaften mit einem Ansatz mit sehr hoher Auflösung als auch (b) Partikeleigenschaften $\mathrm{zu}$ bestimmen.

Die Partikeleigenschaften sind grundsätzlich von großer Bedeutung, wurden jedoch in Hinblick auf die Erosionsproblematik von Turbinen bisher relativ wenig untersucht. Wesentliche
Faktoren in diesem Zusammenhang sind die Kantigkeit (Bahadur und Badruddin 1990) und die Härte (Goodwin et al. 1969) von Partikeln (sog. ,acceleration erosive wear"). Beide Erosionsfaktoren wurden bereits in numerische Modellierungsansätze einbezogen (Stachowiak und Batchelor 1987). Darüber hinaus haben Stachowiak und Batchelor (1987) angeführt, dass das Verhältnis der Teilchenhärte zur Substrathärte der kontrollierende Faktor ist. Zusätzlich quantifizierten empirische Studien den Einfluss der Partikelgröße auf die Modalität und die Rate der erosiven Abnutzung (Hojo et al. 1986).

Es wurde festgestellt, dass Partikelgrößen über 0,2 bis $0,25 \mathrm{~mm}$ besonders schädlich sind. Ab einer Größe von über $0,25 \mathrm{~mm}$ verursachen Partikel selbst bei einer Härte von weniger als 5 auf der Mohs'schen Skala Verschleiß (Neopane 2010). Feine Sedimente bergen ein hohes Risiko für die Erosion von Turbinen, wenn die Turbine unter hohem Druck arbeitet (Padhy und Saini 2011). Wellinger und Utez (1955) und Worster und Denny (1955) beschrieben, dass Abrasion infolge von sog. "sliding“ und sog. „grazing“ direkt proportional zur Größe, jedoch unabhängig von der Größe des direkten Aufpralls ist.

Die Partikelgröße kann hauptsächlich in zwei Grundmaßen - Masse und Länge - charakterisiert werden. Für eine gegebene Geschwindigkeit ist die kinetische Energie des Teilchens direkt proportional zur Masse und die Masse des kugelförmigen Teilchens ist proportional zum Durchmesser (Neopane 2010). Quantitativ können Partikel aufgrund visueller Merkmale als rund, eckig und halbrund charakterisiert werden (Bahadur und Badruddin 1990). In den meisten erosiven Modellen wurden Formparameter (Neopane 2010) mit einigen zusätzlichen Ansät- zen zur Beschreibung der Rauheit mit mehreren Skalen (Drolon et al. 2000) aufgenommen. Darüber hinaus wurde angegeben, dass die Erosion auch direkt proportional zur Härte und Konzentration der Partikel (unabhängig von der Größe) ist (Neopane 2010). Hier haben Labortests für verschiedene Materialien gezeigt, dass der Verschleiß schnell zunimmt, sobald die Partikelhärte die des Turbinenmaterials übersteigt (Stauffer 1958).

Die Wissensdefizite in Bezug auf Turbinenabrasion können wie folgt aufgelistet werden:

i. Es gibt derzeit keinen Überblick über Sediment- und Turbinenabrasion an großen Fließgewässern, die weltweit verfügbar sind, und es wurden noch keine detaillierten (datenbankbasierten) Analysen zu diesem Thema durchgeführt (Abb. 6).

ii. Einer der Hauptnachteile besteht darin, dass sich die Forschung bisher an Sedimentturbinen hauptsächlich auf Turbineneigenschaften (Beschichtung) konzentriert hat, wobei technische Konzepte (Lösungen) zur Minderung von Wechselwirkungen zwischen Turbinen und Sediment nicht berücksichtigt wurden (Hauer et al. 2016b). Darüber hinaus wurde nur sehr wenig geforscht, um die Konstruktion von erosionsbeständigen Strömungskanälen (Neopane 2010) in Bezug auf die variablen Sedimentationseigenschaften von Speicheranlagen zu entwickeln.

iii. Es fehlen vergleichbare Datensätze bezüglich der Sedimenttransportdynamik (Geschiebe, Schwebstoffe) von großen Flusssystemen (globale Sicht), die für die Wasserturbinenindustrie von Interesse sind. 


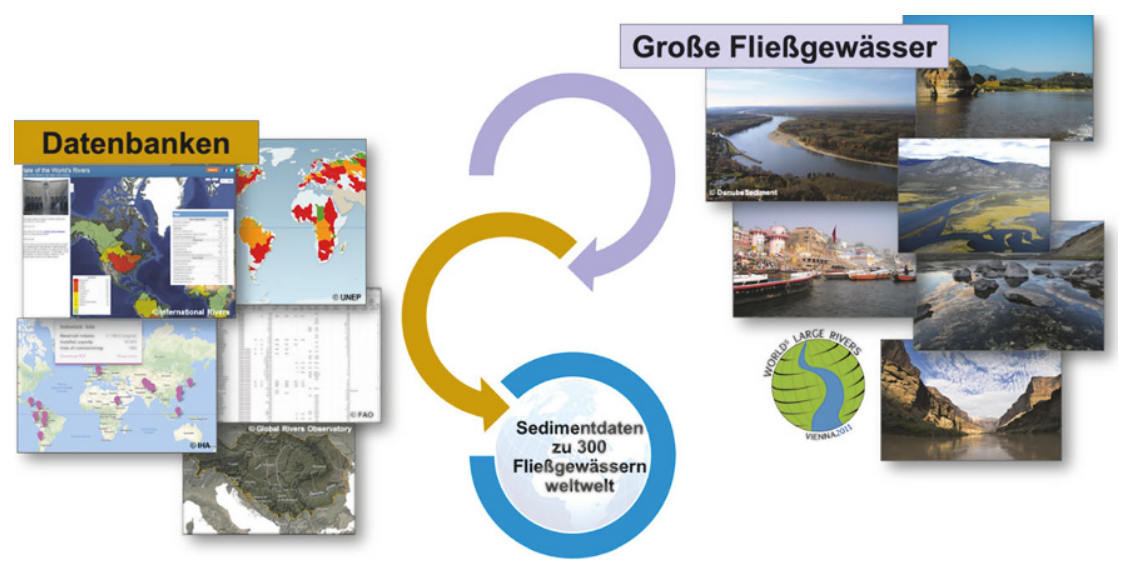

Abb. 6 Erhebung von Sedimentdaten an großen Fließgewässern weltweit

iv. Zusätzlich zum Abrieb der Turbine mangelt es an Wissen zu wirtschaftlichen und technischen Auswirkungen infolge von Stauraumverlandung durch Sedimentation unter Berücksichtigung eines negativ beeinflussten Turbinenbetriebs (Hauer et al. 2016a).

v. Darüber hinaus bestehen Wissensdefizite in Bezug auf ein nachhaltiges Sedimentmanagement bei Wasserkraftwerken zur Optimierung der Langlebigkeit von Turbinen (Hauer et al. 2016a, 2016b).

\section{Abrasion in Sediment-Bypass- Systemen}

Die Sedimentation von Speicheranlagen ist ein zunehmendes Problem für den nachhaltigen Betrieb und die Bewirtschaftung verschiedener hydraulischer Anlagenteile. Eine mögliche Gegenmaßnahme auf Einzugsgebietsbzw. Stauraumebene ist der Einbau von Sediment-Bypass-Systemen, die Sedimente vom stromaufwärts gelegenen Fließgewässer um einen Damm herum bis zum Unterwasserbereich führen. Sediment-Bypass-Systeme tragen dazu bei, die Sedimentanreicherung im Speicher zu verringern und die Sedimentkontinuität im Fließgewässernetz zu erhöhen (Fukuda et al. 2012). Sediment-Bypass-Tunnel zeichnen sich durch hohe Transportkapazitäten (Umleitung des Flusssediments) aus, die durch eine hydraulisch optimierte Ausleitung, gefolgt von einer steilen Tunnelkonstruktion erreicht werden. Daher sind bei Bypass-Systemen hohe Fließgeschwindigkeiten bei schießendem Abfluss (Froude-Zahl $>1$ ) häufig und erzeugen in Kombination mit intensi- ven Sedimenttransportraten einen hohen Abrieb des Beschichtungsmaterials der Tunneloberfläche. Ein wirtschaftlicher Betrieb (geringer Wartungsaufwand) von Sediment-Bypass-Tunneln beruht somit auf einer optimierten Auswahl von Beschichtungsmaterialien, wie beispielsweise Faserbeton oder Granit (Hagmann et al. 2012). Trotz Fortschritten im Bereich der Beschichtungstechnik besteht nach Boes (2015) weiterhin Forschungsbedarf betreffend der Abriebfestigkeit vieler derzeit verwendeter Beschichtungsmaterialien, insbesondere bei einem intensiven Sedimenttransport.

Abrieb ist definiert als kontinuierlicher Materialverlust auf der Oberfläche eines Festkörpers, der durch mechanische Beanspruchung durch Kontakt mit einem festen, flüssigen oder gasförmigen Partikeln verursacht wird (DIN 50320). Nach Helbig und Horlacher (2007) hängt die Abriebspannung in Fluid-Feststoff-Gemischen von (i) Korngröße und Kornverteilung, (ii) Korngeometrie und Kornhärte sowie (iii) hydraulischer Kraft (z. B. Fließgeschwindigkeit) ab. Darüber hinaus wird die Abriebintensität auch von der Transportart (Gleiten, Rollen oder Hüpfen) und der Transportgeschwindigkeit bestimmt, die beide von den hydraulischen Bedingungen abhängen (Auel 2014). Eine Abriebanalyse unter Verwendung eines Sedimentzuführungssystems in einem Laborexperiment, das mit verschiedenen Materialien beschichtet ist, wurde von Auel (2014) durchgeführt. Das Modelldesign basierte auf dem Sediment-Bypass-System am Speicher Solis (Schweiz). Es wurde herausgefunden, dass das Abriebmuster gut mit der gemessenen Sohlschubspannung übereinstimmt und die $\mathrm{Ab}$ - riebmasse linear mit der transportierten Sedimentmasse zunimmt.

In-situ-Messungen der Abriebraten (z. B. unter Verwendung von Fotogrammetrie oder 3D-Lasertechniken) werden häufig in Sedimenttunneln durchgeführt, um den Wartungsvorgang zu planen und zu optimieren (Jacobs und Hagmann 2015). Um jedoch die Beständigkeit gegen Abrieb in hydraulischen Systemen bestimmen zu können, sind genaue Informationen $\mathrm{zu}$ Sedimenttransportgeschwindigkeit und -modus erforderlich - Daten, die in solchen Anlagen in der Regel nicht zur Verfügung stehen. Ein erster Schritt in Richtung eines exakten Sedimenttransportmonitorings in einem Sediment-Bypass wurde durch die Installation eines Geophonsystems (akustisches Sedimentmonitoring) im Sediment-Bypass Solis (Hagmann et al. 2015) erreicht, obwohl detaillierte Ergebnisse bezüglich des Verhältnisses zwischen den beiden gemessenen Parametern Transportrate und Umkehrabrieb noch nicht veröffentlicht wurden.

Derzeit bestehen folgende Wissensdefizite:

i. Standardisierte Auswahlkriterien für Materialien, die in SedimentBypass-Systemen verwendet werden, sind noch nicht verfügbar (Hagmann et al. 2012).

ii. Zur Messung des Abriebs wurden verschiedene Labormethoden entwickelt. Die meisten dieser Methoden sind jedoch bei der Simulation des Transportmodus auf natürliche Fließgewässer begrenzt. Die Übertragbarkeit von Testergebnissen und die Transformation unter In-situ-Bedingungen ist daher in den meisten Fällen nur bedingt realisierbar (Helbig und Horlacher 2007).

iii. Strömungsuntersuchungen zum Abrieb sind in der Modellskala in der Regel begrenzt (z. B. maximale Strömungsgeschwindigkeit, Begrenzungen der Korngröße). Weiters müssen Skalierungseffekte der gesammelten Ergebnisse im Vergleich zu natürlichen Fließgewässern berücksichtigt werden (Auel 2014).

iv. In-situ-Messungen des Abriebs sind selten. Zudem liegen in der Regel keine komplementären Daten zur Abriebspannung (z. B. Sedimenttransportrate, Transportmodus, GSD) vor (Boes 2015). 

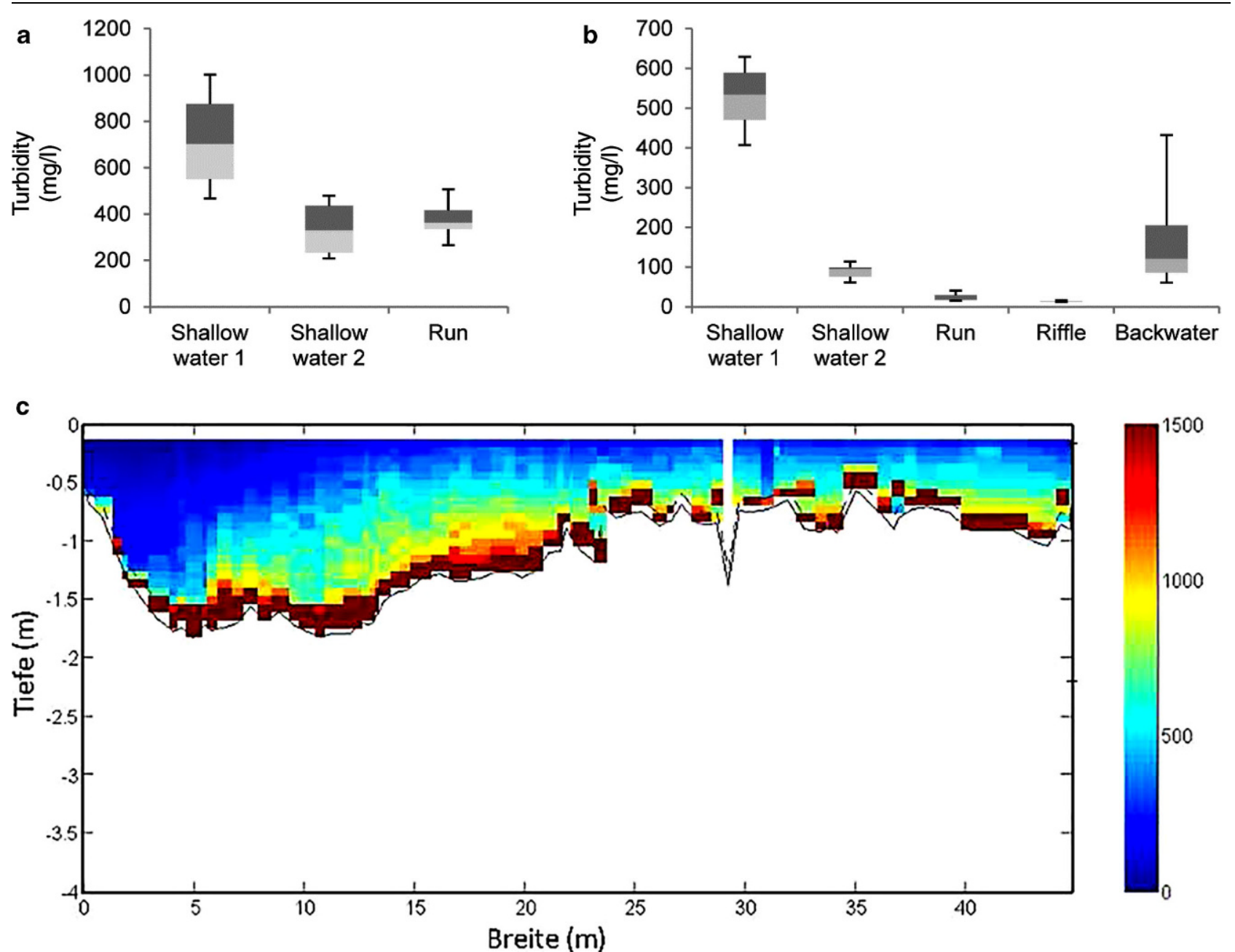

Abb. 7 Habitatbezogene Variation der Trübung an verschiedenen Standorten und Messzeitpunkten während der kontrollierten Absenkung des Gepatsch-Speichers

v. Sediment-Bypass-Systeme sind im Allgemeinen nur während eines Hochwasserereignisses aktiv (Hagmann et al. 2015). Somit ist die Anzahl möglicher Ereignisse zum Messen des Abriebs und der Abriebspannung (z. B. Sedimenttransportrate, Transportmodus, GSD) begrenzt.

\section{Sedimente und Ökologie}

Hydrologie, Sedimentologie und folglich Flussmorphologie bilden das „Rückgrat" unserer Fließgewässer (Maddock 1999). Neben den Elementen der Wasserqualität ist die hydromorphologische/sedimentologische Degradierung der Flusssysteme eine der Hauptbelastungen der Gewässerökologie (Dudgeon et al. 2006). In der EU-Wasserrahmenrichtlinie (Richtlinie 2000/60/EG) werden verschiedene Aspekte hydromorphologischer Störungen erwähnt, die von den europäischen Staaten in Bewirtschaftungsplänen behandelt werden müssen, um die Ziele eines guten ökologischen Zustands oder eines guten ökologischen Potenzials bis 2015 zu erreichen (mit der Möglichkeit einer Verlängerung bis 2027) (Artikel 3 und 4). Um den ,guten ökologischen Zustand“ zu erzielen, ist jedoch der Zustand der Sedimente bzw. die Sedimentdynamik in einem Fließgewässer (z.B. Sedimentkontinuum) nur indirekt Teil der Bewertung. Für den ,guten ökologischen Zustand“ wird hier davon ausgegangen, dass die biotischen Kriterien (Abundanz/Biomasse von Fischen und Makroinvertebraten) den hydromorphologischen Status (Richtlinie 2000/60/EG) widerspiegeln. Dabei werden wesentliche dynamische, sedimentologische Prozesse sowohl mit- tel- als auch langfristig nicht bewertet (Hauer 2015).

Im Allgemeinen spielen Sedimente eine entscheidende Rolle für die Qualität des lokalen physischen Lebensraums. Nach Leopold et al. (2012) gibt es 8 Variablen, die für die morphologische Charakterisierung eines Fließgewässers verantwortlich sind: (i) bordvolle Breite, (ii) bordvolle Tiefe, (iii) Strömungsgeschwindigkeit, (iv) Abfluss, (v) Energieliniengefälle, (vi) Rauigkeit, (vii) Feststofffrachten und (viii) Korngrößen. Darüber hinaus bestimmen die morphologischen Merkmale eines Fließgewässers die Art und Zusammensetzung seiner Lebensräume. Störungen einer dieser Variablen könnten den morphologischen Typ des Flusses und folglich die Zusammensetzung des Lebensraums verändern. Sedimente sind sowohl lebensraumbildende Elemente (z.B. Felsbrocken) 
als auch Teil morphologischer Strukturen (Kies an Schotterbänken) (Hauer et al. 2014). Mögliche Auswirkungen von Sedimentstörungen auf aquatische Organismen können in Bezug auf die zeitliche Komponente wie auch in Bezug auf die Wirkungsweise des Einflusses (direkt oder indirekt) unterschieden werden (Hauer et al. 2013). Zum einen gibt es mittel- bis langfristige, indirekte Auswirkungen aufgrund von Änderungen der physikalischen Umgebung (z. B. Änderungen in der Sedimentologie, Verlust von Laichplätzen; siehe habitatbezogene Variation der Trübung während kontrollierter Stauraumabsenkungen, Abb. 7) und zum anderen existieren kurzfristige, direkte (hohe dynamische) Auswirkungen aufgrund von physiologischem Stress (z.B. hohe Trübung für Fische infolge von Stauraumspülungen) oder Abriebgefahr (z. B. für Makroinvertebraten).

Die Störung des Sedimentregimes hängt mit Sedimentdefiziten oder -überschüssen und dem Sedimenttransport zusammen (z. B. Sutherland et al. 2002). Insbesondere in alpinen Regionen sind die Auswirkungen von Sedimentdefiziten für die teilweise und kontinuierlich fortschreitende Eintiefung der Fließgewässerstrecken und die damit verbundene Verschlechterung des Habitats verantwortlich (Habersack und Piégay 2007). Eine künstliche Verringerung des Sedimentinputs (z. B. Wildbachsperren, Wasserkraftnutzung) kann zwei Formen der Auswirkungen auf die Korngrößenzusammensetzung haben: Einerseits führt die Vergröberung des Substrats durch selektiven Transport in Abhängigkeit von der Hochwasserhäufigkeit zur Abpflasterung der Gewässersohle (Hauer 2015), andererseits kann sich in alpinen Becken mit feinen Materialablagerungen aus dem Tertiär (Meeresablagerungen) unterhalb der quartären Kiesschicht die Gefahr eines sogenannten "Sohldurchschlags“ (Habersack und Klösch 2012) entwickeln (z.B. an der Salzach im Jahr 2002). Die Folgen von Sedimentdefiziten und Auswirkungen auf das Fließgewässer sind (i) Abnahme der Heterogenität des Lebensraums (Kondolf 1997; Hauer et al. 2014), (ii) negative Auswirkungen auf Uferstrukturen (Rinaldi und Casagli 1999), (iii) Gefährdung von Bauwerken, wie z.B. Risiko betreffend die Stabilität von Brückenpfeilern (Hauer et al. 2006), (iv) Risiko von Verwerfungen bei extremen Ereignissen (Brizga und Finlayson 1990; Krapesch et al. 2011) und (v) Abnah- me von Sedimenttransportraten sowie der flusstypspezifischen Sedimentqualität (Kondolf 1997), insbesondere von wesentlicher Bedeutung für Laichhabitate salmonider Fischarten (Hauer et al. 2014).

In Bezug auf ökologische Auswirkungen können u. a. folgende Wissensdefizite abgeleitet werden:

i. Fehlen von Trübungsanalysen für verschiedene Flusssysteme (bezogen auf den Lebensraum),

ii. Mangel an wissenschaftlich begründeten Methoden zur Bewertung der Auswirkungen von Sedimentmanagementmaßnahmen auf die Gewässerökologie,

iii. unzureichendes Wissen zu Wechselwirkung von Sedimentkonzentration/Trübung mit aquatischen Organismen,

iv. Fehlen eines tragbaren objektiven Messsystems zur Analyse des Konsolidierungszustands aus ökologischer Sicht (z.B. Kolmation von Laichplätzen) und

v. unzureichende Kenntnisse über den potenziellen Abrieb von Wasserorganismen und Pflanzen sowie $\mathrm{zu}$ Wechselwirkungen zwischen Biofilm/Makrophyten und Sedimentstabilisierung.

\section{Zusammenfassung und Ausblick}

Die Vielfalt an Herausforderungen im Zuge des Sedimentmanagements bei Wasserkraftanlagen, aber auch bei Fließgewässern im Allgemeinen, zeigt die Notwendigkeit, in diesem mehrjährigen Forschungsprogramm gemeinsam mit der Wirtschaft, VertreterInnen der Behörden und der Wissenschaft eine nationale, standardisierte Vorgehensweise $\mathrm{zu}$ entwickeln. In einem mehrstufigen Verfahren der Umsetzung des CD-Labors wird in den ersten beiden Jahren 2017 bis 2019 Grundlagenforschung in Bezug auf ein verbessertes Prozessverständnis des Sedimenttransports durchgeführt. Weiters werden in wasserbaulichen Versuchen Fragen zur Turbulenzskalierung ebenso wie zur Remobilisierung von kohäsivem und konsolidiertem Feinmaterial ein wesentlicher Teil des Versuchsprogramms sein. Anwendung und Weiterentwicklung von neuen Messtechnologien bilden in dieser ersten Phase ebenfalls einen bedeutenden wissenschaftlichen Schwerpunkt. In Phase 2 (2019 bis 2022) des CD-Labors „Sedimentforschung und -management“ ist geplant, die ökologischen Untersuchungen auszuweiten, um einige der wesentlichen Fragen bzw. existierenden Wissensdefizite zu beantworten. Parallel dazu, aber vor allem auch in weiterer Folge sind Abstimmungen mit den Behörden (Ministerium) in Hinblick auf den Nationalen Gewässerbewirtschaftungsplan 3 wesentlich. $\mathrm{Zu}$ einem künftigen Zeitpunkt des CDLabors wird auch die Einbindung des neuen Wasserbaulabors an der BOKU mit großmaßstäblichen Versuchen erfolgen. Hier ist geplant, vor allem die bekannten Phänomene und Probleme betreffend die Skalierung von physikalischen Versuchen $\mathrm{zu}$ verbessern und in großmaßstäblichen Versuchen $\mathrm{Op}$ timierungen von Wasserkraftanlagen durchzuführen.

Somit bilden die in diesem Fachartikel beschriebenen fachlichen Komponenten des CD-Labors eine Vielzahl von Aspekten, die sowohl eine ökonomische und technische, aber auch ökologische Optimierung ermöglichen. Vor allem unter einem der Leitsätze „Flüsse wieder fit für mehr Sediment“ - werden auch flussbauliche Fragen im Detail beantwortet, um letztendlich klare Aussagen zu bekommen, wie die gewässermorphologischen Rahmenbedingungen sein müssen, damit ein verbessertes Sedimentkontinuum (Durchgängigkeit) auch zu einer Verbesserung der morphologischen Situation führt (z.B. dynamische Umlagerungstrecken mit hoher Habitatheterogenität). Unter diesem angeführten Leitsatz wird auch die auf wissenschaftlichen Untersuchungen und Ergebnissen basierende Diskussion geführt werden, die existierende "Zweiklassengesellschaft" in Sedimentfragen gesamtheitlich $\mathrm{zu}$ betrachten. Es gibt in natürlichen Gewässersystemen keine klare Zuordnung. welche Feststoffe positiv (z. B. Geschiebe) und welche als negativ (z. B. Feinsediment) anzusehen sind. Hier wird die anwendungsorientierte Grundlagenforschung klare Aussagen ermöglichen. Zusammenfassend sind es große Herausforderungen, die in den nächsten Jahren auf Forschung und Wirtschaft in Fragen des Sedimentmanagements zukommen werden, wobei das in diesem Fachbeitrag beschriebene CD-Labor "Sedimentforschung und -management" ein wesentlicher Bestandteil der Lösungsfindung sein wird.

Danksagung Wir bedanken uns für die finanzielle Unterstützung durch 
die Christian Doppler Forschungsgesellschaft, das Bundesministerium für Digitalisierung und Wirtschaftsstandort und die Nationalstiftung für Forschung, Technologie und Entwicklung. Darüber hinaus möchten wir unseren Unternehmenspartnern Verein für Ökologie und Umweltforschung (VÖU), viadonau, Andritz AG und Voith $\mathrm{GmbH}$ für die
Förderung unserer Forschungsarbeiten im Rahmen des CD-Labors danken.

Funding Open access funding provided by University of Natural Resources and Life Sciences Vienna (BOKU).

Open Access Dieser Artikel wird unter der Creative Commons Namensnennung 4.0 International Lizenz (http:// creativecommons.org/licenses/by/4 $0 /$ deed.de) veröffentlicht, welche die Nutzung, Vervielfältigung, Bearbeitung, Verbreitung und Wiedergabe in jeglichem Medium und Format erlaubt, sofern Sie den/die ursprünglichen $\mathrm{Au}$ tor(en) und die Quelle ordnungsgemäß nennen, einen Link zur Creative Commons Lizenz beifügen und angeben, ob Änderungen vorgenommen wurden.

\section{Literatur}

Agrawal, Y., Mikkelsen, O. A, Pottsmith, H. C (2011): Sediment Monitoring technology for turbine erosion and reservoir siltation application, Proceedings of HYDRO 2011 Conference, Prague, Czech Republic.

An, S., Julien, P. (2014): Three-Dimensional Modeling of Turbid Density Currents in Imha Reservoir, South Korea, Journal of Hydraulic Engineering, 140(5), 05014004.

Ashmore, P. E., Rennie, C. D. (2013): Gravel-bed rivers: from particles to patterns, Earth Surface Processes and Landforms, 38(2), 217-220.

Auel, C. (2014): Flow characteristics, particle motion and invert abrasion in sediment bypass tunnels, Dissertation at the ETH Zurich, Switzerland, Nr. 22008.

Auel, C., Boes, R. M., Ziegler, T., Oertli, C. (2011): Design and construction of the sediment bypass tunnel at Solis, The International Journal on Hydropower \& Dams, 13(3), 62-66.

Bahadur, S., Badruddin, R. (1990): Erodent particle characterization and the effect of particle size and shape on erosion, Wear, 138(1), 189-208. Boes, R. (2011): Nachhaltigkeit von Talsperren angesichts der Stauraumverhandlung, 41. Internationales Wasserbau-Symposium, Aachen, Germany.

Boes, R. (2015): First International Workshop on Sediment Bypass Tunnels, In: Boes, R. (Eds.) First International Workshop on Sediment Bypass Tunnels, Zurich, Laboratory of Hydraulics, Hydrology and Glaciology (VAW), ETH Zurich, Switzerland.

Brizga, S. O., Finlayson, B. L. (1990): Channel avulsion and river metamorphosis: The case of the Thomson River, Victoria, Australia Earth Surface Processes and Landforms, 15(5), 391-404.

De Cesare, G., Boillat, J. L., Schleiss, A. J. (2006): Circulation in stratified lakes due to flood-induced turbidity currents, Journal of Environmental Engineering, 132(11), 1508-1517.

Drolon, H., Druaux, F, Faure, A. (2000): Particles shape analysis and classification using the wavelet Transform, Patterm Recognition Letters, 21(6-7), 473-482.

Dudgeon, D., Arthington, A. H., Gessner, M. O. Kawabata, Z. I., Knowler, D. J., Lévêque, C., ... Sullivan, C. A. (2006): Freshwater biodiversity: importance, threats, status and conservation challenges. Biological Reviews, 81(2), 163-182.

European Commission (2018): 2050 long term strategy. URL: https://ec.europa.eu/clima/ policies/strategies/2050_en (accessed: 20.12. 2018)

Feng, L., Qingshu, Y., Shenliang, C., Luo, Z. F, Yuan, F., Wang, R. T. (2014): Temporal and spatial variability of sediment flux into the sea from the three largest rivers in China, Journal of Asian Earth Sciences, 87, 102-115.

Fukuda, T., Yamashita, K, Osada, K., Fukuoka, S. (2012): Study on Flushing Mechanism of Dam Reservoir Sedimentation and Recovery of Riffle-Pool in Downstream Reach by a Flushing
Bypass Tunnel, Proceedings of the International Symposium on DAMS FOR A CHANGING WORLD-Need for Knowledge Transfer across the Generations \& the World, Kyoto, Japan.

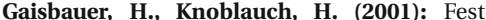
stoffmanagement bei Stauanlagen. Österreichische Wasser- und Abfallwirtschaft, 53(11-12), 265-268.

Glas, M., Tritthart, M., Zens, B., Keckeis, H., Hauer, C., Habersack, H. (2012): Larval dispersal patterns: observations and particle tracing simulations in a racetrack flume, Proceedings des 9th International Symposium on Ecohydraulics, 17-21 September, Wien, Österreich.

Goodwin, J. E., Sage, W., Tilly, G. P. (1969): Study of Erosion by Solid Particles, Proceedings of the Institution of Mechanical Engineers, 184(1) 279-292.

Habersack, H., Klösch, M. (2012): Monitoring und Modellierung der Ufererosion, Interim Report of the Institute of Water Management, Hydrology and Hydraulic Engineering, University of Natural Resources and Life Sciences, Vienna, commissioned by viadonau.

Habersack, H., Piégay, H. (2007): River restoration in the Alps and their surroundings: past experience and future challenges, Developments in Earth Surface Processes, 11, 703-735.

Hagmann, M., Albayrak, I., Boes, R. (2015) Field research: Invert material resistance and sediment transport measurements, In: Boes, R. (Eds). First International Workshop on Sediment Bypass Tunnels, Zürich. Laboratory of Hydraulics, Hydrology and Glaciology (VAW), ETH Zurich, Switzerland.

Hagmann, M., Auel, C., Albayrak, I., Boes, R. M. (2012): Hydroabrasion in Sedimentumleitstollen, Proceedings of the 35th Dresdner Wasserbaukolloquium, TU Dresden, Germany.

Harby, A., Baptist, M., Duel, H., Dunbar, M., Goethals, P., Huusko, A., Ibbotson, A., Mader, H. Pedersen, M. L., Schmutz, S., Schneider, M. (2005): Cost 626 European Aquatic Modelling Network, Proceedings des Final Meeting, Silkeborg, Dänemark, 402.

Hassan, M. A., Church, M., Rempel, J., Enkin, R. J. (2009): Promise, performance and current limitations of a magnetic Bedload Movement Detector, Earth Surface Processes and Landforms, 34(7), 1022-1032.

Hauer, C. (2015): Review of hydro-morphological management criteria on a river basin scale for preservation and restoration of freshwater pearl mussel habitats, Limnologica, 50, 40-53.

Hauer, C., Habersack, H. (2009): Morphodynamics of a 1000-year flood in the Kamp River, Austria, and impacts on floodplain morphology, Earth Surface Processes and Landforms, 34(5), 654-682.

Hauer, C., Frangez, C., Jungwirth, M., Hofbauer, S., Muhar, S., Preis, S., Unfer, G., Habersack, H. (2006): Flussmorphologische Veränderungen am Kamp durch das Hochwasser 2002 und ihre Bedeutung für Hochwasserschutz und Fischöko- logie. Österreichs Wasser- und Abfallwirtschaft, 11-12, 175-183.

Hauer, C., Haimann, M., Holzapfel, P., Leitner,

P., Graf, W., Holzer, G., Habersack, H. (2016a): Monitoring of the lake-emptying of the Gepatsch reservoir / TIWAG. Final report. $164 \mathrm{pp}$.

Hauer, C., Lichneger, P., Holzapfel, P., Flödl, P. (2016b): Monitoring of a residual flow turbine prototype for an improved sediment continuum. Mid-term evaluation report.

Hauer, C., Schober, B., Habersack, H. (2013). Impact analysis of river morphology and roughness variability on hydropeaking based on numerical modelling, Hydrological Processes, 27(15), 2209-2224

Hauer, C., Unfer, G., Holzapfel, P., Haimann, M., Habersack, H. (2014): Impact of channel bar form and grain size variability on estimated stranding risk of juvenile brown trout during hydropeaking, Earth Surface Processes and Landforms, 39(12), 1622-1641.

Haun, S., Dorfmann, C., Harb, G., Olsen, N. R. B. (2012): Proceedings of the IAHR European Conference, Munich, Germany.

Helbig, U., Horlacher, H. B. (2007): Ein Ansatz zur versuchstechnischen Simulation und Übertragbarkeit der Hydroabrasion strömungsbeanspruchter Betonoberflächen im Wasserbau, Bautechnik, 84, 641-645.

Hojo, H., Tsuda, K., Yabu, T. (1986): Erosion damage of polymeric material by slurry, Wear, 112(1), 17-28.

Jacobs, F., Hagmann, M. (2015): Sediment bypass tunnel Runcahez: Invert abrasion 1995-2014, In: Boes, R. (Eds.) First International Workshop on Sediment Bypass Tunnels 2015 Zürich, ETH Zurich, Switzerland.

Kantoush, S. A., Schleiss, A. J. (2009): Channel formation during flushing of large shallow reservoirs with different geometries, Environmental Technology, 30, 855-863.

Kondolf, M. (1997): Hungry Water: Effects of Dams and Gravel Mining on River Channels, Environmental Management, 21(4), 533-551. Kondolf, G. M., Gao, Y., Annandale, G. W., Morris, G. L., Jiang, E., Zhang, J., Cao, Y., Carling, P., Fu, K., Guo, Q., Hotchkiss, R., Peteuil, C., Sumi, T., Wang, H. W., Wang, Z., Wei, Z., Wu, B., Wu, C., Yang, C. T. (2014): Sustainable sediment management in reservoirs and regulated rivers: $\mathrm{Ex}-$ periences from five continents, Earth's Future, 2, 256-280.

Krapesch, G., Hauer, C., Habersack, H. (2011) Scale orientated analysis of river width changes due to extreme flood hazards, Natural Hazards and Earth System Sciences, 11(8), 2137-2147.

Leopold, L. B., Wolman, M. G., Miller, J. P. (2012): Fluvial processes in geomorphology. Courier Corporation, New York.

Lin, P., Liu, P. L. F. (1998): A numerical study of breaking waves in the surf zone, Journal of Fluid Mechanics, 359(1), 239-264.

Lysne, D. K., Glover, B., Støle, H., Tesaker, E. (2003): Hydraulic design, Norwegian Univer- 
sity of Science and Technology, Department of Hydraulic and Environmental Engineering, Trondheim.

Maddock, I. (1999): The importance of physical habitat assessment for evaluating river health, Freshwater biology, 41(2), 373-391.

Mamede, G. L. (2008): Reservoir Sedimentation in Dryland Catchments: Modelling and Management, Dissertation for the Degree of Doctor of Natural Sciences at the Faculty of Mathematics and Sciences at the Universität Potsdam, Deutschland.

Monaghan, J. J., Kos, A. (1999): Solitary waves on a Cretan beach. Journal of waterway, port, coastal, and ocean engineering, 125(3), 145-155.

Neopane, H. P. (2010): Sediment Erosion in Hydro Turbines, Dissertation at the Faculty of Engineering Science and Technology, Norwegian University of Science and Technology.

Padhy, M. K., Saini, R. P. (2009): Effect of size and concentration of silt particles on erosion of Pelton turbine buckets, Energy, 34(10), 1477-1483. Padhy, M. K., Saini, R. P. (2011): Study of silt erosion on the performance of a Pelton turbine, Energy, 36, 141-147.

Patankar, S. V., Spalding, D. B. (1972): A Calculation Procedure for Heat, Mass and Momentum Transfer in Three-Dimensional Parabolic Flows, International Journal of Heat and Mass Transfer, 15, 1787-1806.

Rinaldi, M., Casagli, N. (1999): Stability of streambanks formed in partially saturated soils and effects of negative pore water pressures: the Sieve River (Italy), Geomorphology, 26, 253-277.
Schleiss, A., Oehy, C. (2002): Verlandung von Stauseen und Nachhaltigkeit, Wasser Energie Luft - eau énergie air, 94 (7/8), 227-234. Schleiss, A., De Cesare, G., Jenzer Althaus, J. (2010): Verlandung der Stauseen gefährdet die nachhaltige Nutzung der Wasserkraft, Wasser Energie Luft, 102(1), 31-40.

Schlüter, J. U., Wu, X., Kim, S., Shankaran, S., Alonso, J. J., Pitsch, H. (2005): A framework for coupling Reynolds-averaged with large-eddy simulations for gas turbine applications, Journal of Fluids Engineering, 127(4), 806-815. Stachowiak, G. W., Batchelor, A. W. (1987): Dimensional Analysis Modelling Tribological Data International Tribology Conference 1987, Melbourne, 2-4 December 1987, Preprints of Papers, 255.

Stauffer, W. A. (1958): The abrasion of hydraulic plant by sandy water. Schweizer Archiv Für Angew. Wiss. Technik, 24 (7/8), 3-30.

Sumi, T., Okano, M., Takata, Y. (2004): Reservoir sedimentation management with bypass tunnels in Japan, Proceedings des 9th International Symposium on River Sedimentation, Yichang, China, 1036-1043.

Sutherland, A. B., Meyer, J. L., Gardiner, E. P. (2002): Effects of land cover on sediment regime and fish assemblage structure in four southern Appalachian streams, Freshwater Biology, 47(9), 1791-1805.

Thapa, B. S., Thapa, B., Dahlhaug, O. G. (2012): Empirical modelling of sediment erosion in Francis turbines, Energy, 41(1), 386-391.
Tritthart, M. (2000): Anwendung von dreidimensionalen numerischen Methoden beim Sedimentmanagement in Talsperrenreservoirs, Master Thesis an der Universität Innsbruck, Österreich.

Tritthart, M., Schober, B., Habersack, H. (2011): Non-uniformity and layering in sediment transport modelling 1: flume simulations, Journal of Hydraulic Research, 49(3), 325-334.

Truscott, G. F. (1972): A literature survey on abrasive wear in hydraulic machinery, Wear, 20(1), 29-50.

Wagner, B., Habersack, H., Hauer, C., Schoder, A. (2013): Sedimentmanagement bei Wasserkraftwerken, Wasserkraft, 39, 10-11.

Wellinger, K., Utez, H. (1955): Sliding scouring and blasting wear under the influence of granular of solids. Vdi-Forschungsheft, 21b, 449.

Worster, R. C., Denny, D. F. (1955): Hydraulic transport of solid material in pipes, Proceedings of the Institution of Mechanical Engineers, 169(1), 563-586.

Young, D., Lin, Q., Murugesan, K. (2005): Twodimensional simulation of a thermally stratified reservoir with high sediment-laden inflow, Journal of Hydraulic Research, 43(4), 351-365.

Yu, W. S., Lee, H. Y., Hsu, S. M. (2000): Experiments on deposition behavior of fine sediment in a reservoir, Journal of Hydraulic Engineering, $126,912-920$. 